and L-cysteine in medulla were determined by UPLC-MS/MS Malondialdehyde (MDA) in medulla was determined by HPLC.

Results Reduced glutathione (GSH) was significantly reduced in the brainstem of rat pups submitted to chronic intermittent hypoxic episodes associated with reduction in GSH/GSSG ratio. GSH precursors, -glutamyl-cysteine and L-cysteine were also significantly lower in the brainstem of intermittent hypoxia group.

\section{INTERACTION OF INFLAMMATION AND HYPEROXIA IN NEONATAL WHITE MATTER DAMAGE}

doi:10.1136/archdischild-2012-302724.0313

${ }^{1} \mathrm{~F}$ Brehmer, ${ }^{2} \mid \mathrm{Bendix},{ }^{3.4 Y}$ van de Looij, ${ }^{5} \mathrm{M}$ Sifringer, ${ }^{3} \mathrm{~S}$ Sizonenko, ${ }^{6} \mathrm{C}$ Mallard, ${ }^{1} \mathrm{C}$ Bührer, ${ }^{2} U$ FelderhoffMüser, 'B Gerstner. 'Neonatology, Charité Universitätsmedizin Berlin, Berlin; 2Department of Pediatrics 1, Neonatology, University Hospital Essen, Essen, Germany; ${ }^{3}$ Department of Pediatrics, University of Geneva, Geneva: ${ }^{4}$ Laboratory of Functional and Metabolic Imaging, Ecole Polytechnique Fédérale de Lausanne, Lausanne, Switzerland; ${ }^{5}$ Department of Anesthesiology and Intensive Care Medicine, Charité Universitätsmedizin Berlin, Berlin, Germany; ${ }^{6}$ Physiology, University of Gothenburg, Gothenburg, Sweden; 'Paediatric Heart Center, University Hospital Giessen, Giessen, Germany

Intrauterine infection/inflammation are major causes of preterm birth. The dramatic rise of oxygen tissue tension compared to intrauterine conditions amounts to relative hyperoxia in preterm infants. Both, infection/inflammation and hyperoxia have been shown to be involved in brain injury of preterm infants. Hypothesizing an additive or synergistic effect, we investigated the influence of a systemic lipopolysaccharide (LPS) application on hyperoxia-induced white matter damage (WMD) in newborn rats.

Three-day-old Wistar rat pups received $0.25 \mathrm{mg} / \mathrm{kg}$ LPS i.p. and were subjected to $80 \%$ oxygen on P6 for $24 \mathrm{hrs}$. WMD was assessed by immunohistochemistry, western blot and diffusion tensor magnetic resonance imaging. In addition, LPS and hyperoxia were studied in an in vitro co-culture system of primary rat oligodendrocytes and microglia cells. Both noxious stimuli, hyperoxia and LPS, induced a significant increase in apoptotic cell death as revealed by elevatation of cleaved caspase-3 and TUNEL-positive cells. Furthermore, both hyperoxia and LPS caused hypomyelination, as revealed by western blot, immunohistochemistry and altered WM microstructure on MRI. However, the combination of hyperoxia and LPS did neither increase nor decrease cell death and hypomyelination in vivo. In contrast, LPS pre-incubation reduced oligodendrocyte susceptibility towards hyperoxia in vitro.

Our data suggest that inflammation and hyperoxia strongly attenuate oligodendrocyte maturation by apoptotic and nonapoptotic pathways. If both insults are combined, second phase releases of protective cytokines can partially prevent oligodendrocyte cell death. The knowledge of interactions between inflammation and hyperoxia might offer new therapeutic opportunities to prevent $\mathrm{WMD}$ in preterm infants.

\section{HYPEROXIA CHANGES THE BALANCE OF THE THIOREDOXIN/PEROXIREDOXIN SYSTEM IN THE NEONATAL RAT BRAIN}

doi:10.1136/archdischild-2012-302724.0314

${ }^{1} \mathrm{~K}$ Strasser, ${ }^{1} \mathrm{I}$ Bendix, ${ }^{2} \mathrm{U}$ Weichelt, ${ }^{2} \mathrm{~S}$ Endesfelder, ${ }^{3} \mathrm{C}$ von Haefen, ${ }^{4} \mathrm{R}$ Heumann, ${ }^{4} \mathrm{~A}$ Ehrkamp, ${ }^{2} \mathrm{C}$ Buehrer, 'U Felderhoff-Mueser, ${ }^{2,3} \mathrm{M}$ Sifringer. 'Department of Pediatrics 1. Neonatology, University Children's Hospital, Essen; ${ }^{2}$ Department of Neonatology; ${ }^{3}$ Department of Anaesthesiology and Intensive Care Medicine, Charite Campus Virchow, Berlin; ${ }^{4}$ Department for Molecular Neurobiochemistry, Ruhr-University Bochum, Bochum, Germany

Background and Aim As demonstrated previously, oxygen contributes to the pathogenesis of neonatal brain damage and leading to neurocognitive impairment of prematurely born infants in later life.
Reactive oxygen species (ROS) and intrinsic antioxidant defense systems play an important role in both physiological cell signaling processes and many pathological conditions, including neurodegenerative disorders and oxygen-toxicity. Beside the glutathione-system several other redox-modulating proteins are known to be involved in redox-homeostases. The aim of this study was to evaluate potential alterations within the thioredoxin/peroxiredoxin system after exposures to nonphysiologic high oxygen levels in the developing rat brain.

Methods Six-days old Wistar rats were exposed to $80 \%$ oxygen for $6,12,24$ or 48 hours and littermates kept in room air served as controls $(n=6-8)$. Brains (excluding cerebellum) were evaluated after perfusion with PBS and dissection of both hemispheres for RNA and protein analyses.

Results We demonstrate that elevated oxygen concentrations change the balance of the ROS-dependent thioredoxin/peroxiredoxin system. Oxygen-toxicity significantly induced upregulation of peroxiredoxins in infant rat brain. In parallel, hyperoxia reduced the level of DJ-1, a hydroperoxide-responsive protein.

Discussion These findings are highly relevant from a clinical aspect because oxygen administration to neonates is often inevitable, and we recommend that every effort should be made in neonatal medicine to limit exposure of these immature babies to high oxygen concentrations. These results may also contribute to receive optimal therapeutical approaches to ameliorate oxygen toxicity.

\section{AORTIC AND CAROTID INTIMA-MEDIA THICKENING IN APPROPRIATE-FOR-GESTATIONAL AGE PRETERM NEWBORNS WITH ADEOUATE BUT LOW BIRTH WEIGHT}

doi:10.1136/archdischild-2012-302724.0315

U Schubert. Neonatology, Karolinska University Hospital, Karolinska Institutet, Stockholm, Sweden

Purpose Assessment of intima-media thickness (IMT) of the aorta and carotid artery in appropriate for gestational age (AGA) infants during the first 6 months of life after very preterm birth.

Methods Longitudinal ultrasound assessment including 21 very preterm and 29 infants born at term (all AGA) during a six-months period corresponding to the third trimester of pregnancy and the first 3 months after term, measuring aortic and carotid IMT by an angle-corrected M-Mode, and assessment of blood pressure at final follow-up.

Results No differences in aortic or carotid IMT or blood pressure measurements were found between the two groups. However, in relation to vessel lumen diameter, IMT is significantly higher in both arteries in infants born preterm $(p=0.003$ for aorta and $p=0.001$ for carotid artery).

Conclusion In relation to vessel diameter, infants born preterm show thickening of the intima-media in the great arteries. It remains to be established whether this relative intima-media thickening persists into childhood and may be a risk marker for future cardiovascular disease among subjects born preterm.

\section{EARLY VERSUS DELAYED CYCLOSPORINE TREATMENT IN CARDIAC RECOVERY AND INTESTINAL INJURY DURING RESUSCITATION OF ASPHYXIATED NEWBORN PIGLETS}

doi:10.1136/archdischild-2012-302724.0316

${ }^{1}$ RS Gill, ${ }^{2 T F}$ Lee, ${ }^{3} \mathrm{C}$ Sergi, ${ }^{2} \mathrm{C}$ Joynt, 'DL Bigam, ${ }^{1,2} \mathrm{PY}$ Cheung. ' Surgery; ${ }^{2}$ Pediatrics; ${ }^{3}$ Pathology and Labaratory Medicine, University of Alberta, Edmonton, AB, Canada

Background Recently, we have demonstrated that treating asphyxiated newborn piglets with intravenous cyclosporine immediately following resuscitation can improve cardiac function. However, immediate treatment may not be feasible for a large portion of newborns delivered in peripheral/rural hospitals. Therefore, our 
objective was to determine if delayed cyclosporine treatment was still effective in protecting asphyxiated piglets. We hypothesize that both early and delayed treatment with cyclosporine A would improve cardiac recovery during resuscitation of asphyxiated newborn piglets.

Methods Thirty piglets (1-4 days-old) were instrumented for continuous monitoring. After stabilization, normocapnic alveolar hypoxia (10-15\% oxygen) was instituted for $2 \mathrm{~h}$ followed by reoxygenation for $6 \mathrm{~h}$. Piglets were block-randomized to receive either early (5 min of reoxygenation) or delayed (120 min reoxygenation) intravenous bolus of cyclosporine $(10-\mathrm{mg} / \mathrm{kg})$ or saline (control) at identical times during reoxygenation ( $n=8 /$ group). Myocardial and intestinal lactate concentrations as well as histological examinations were determined.

Results Hypoxic piglets had cardiogenic shock (cardiac output $52 \pm 1 \%$ of baseline), hypotension and acidosis. Although both early and delayed cyclosporine treatment improved cardiac output $(\mathrm{P}<0.05$ vs. controls), only early cyclosporine treatment improved stroke volume and systemic oxygen delivery ( $p<0.05 \mathrm{vs}$. controls). Left ventricle and intestinal lactate were higher in controls than in both cyclosporine-treated groups. Early, but not delayed, cyclosporine treatment also attenuated intestinal injury compared to controls $(\mathrm{P}<0.05)$.

Conclusion This study demonstrates that both early and delayed cyclosporine treatment during resuscitation improves cardiac recovery in asphyxiated newborn piglets. However, early treatment with cyclosporine may offer superior cardioprotection and attenuates $\mathrm{H}-\mathrm{R}$ intestinal injury.

\section{THE CARDIO-PROTECTIVE EFFECTS OF DOXYCYCLINE IN A SWINE MODEL OF NEONATAL ASPHYXIA}

doi:10.1136/archdischild-2012-302724.0317

1J LaBossiere, 'JS Pelletier, 'D Bigam, 1,2PY Cheung. 'Surgery; ${ }^{2}$ Pediatrics and Pharmacology, University of Alberta, Edmonton, AB, Canada

Background Myocardial reoxygenation injury following asphyxia in neonates is common. Matrix metalloproteinase-2 activation is associated with myocardial ischemic-reperfusion injury and stunning. Previous in vitro, ex vivo, and small animal studies have demonstrated the cardio-protective qualities of doxycycline, a known inhibitor of matrix metalloproteinase-2, however, large animal models demonstrating this effect are lacking. We hypothesized that doxycycline would improve cardiac recovery and systemic hemodynamics in asphyxiated newborn piglets.

Methods Piglets (1-5 days old) were acutely instrumented for continuous monitoring of heart rate, cardiac output [CO], mean systemic and pulmonary arterial pressures (SAP and PAP, respectively). After stabilization, $2 \mathrm{hrs}$ of normocapnic alveolar hypoxia (10-15\% oxygen) was induced followed by 4 hrs of normoxic reoxygenation ( $21 \%$ oxygen). Piglets were blindly randomized to receive either normal saline or doxycycline $(3,10$, or $30 \mathrm{mg} / \mathrm{kg}$ ) intravenously 5 minutes into reoxygenation ( $\mathrm{n}=7 /$ group). Sham-operated piglets $(\mathrm{n}=5)$ received no hypoxia-reoxygenation.

Results Asphyxiated piglets demonstrated acidosis ( $\mathrm{pH}=7.04 \pm$ $[\mathrm{SD}] 0.08)$, hypotension $(\mathrm{SAP}=31 \pm 3 \mathrm{mmHg}$ ), and cardiac dysfunction $(\mathrm{CO}=58 \pm 8 \%$ of normoxic baseline). Doxycycline had dose-related improvements in $\mathrm{CO}$ and stroke volume $(30 \mathrm{mg} / \mathrm{kg}: 86 \pm 8 \%$ and $79 \pm 15 \%$ of normoxic baseline vs. $65 \pm 7 \%$ and $50 \pm 13 \%$ in controls, respectively [both $\mathrm{p}<0.05]$ ), with no significant change in heart rate compared to controls. Furthermore, SAP was higher and PAP/SAP ratio was lower than those of controls $(p<0.05)$, with no difference in PAP.

Conclusions In an established swine model of neonatal hypoxiareoxygenation, post-resuscitation administration of intravenous doxycycline improves cardiac recovery with beneficial hemodynamic effects in systemic and pulmonary circulations.

Funded by Canadian Institutes of Health Research (CIHR).

\section{CAN EARLY B-TYPE NATRIURETIC PEPTIDE ASSAYS PREDICT SYMPTOMATIC PATENT DUCTUS ARTERIOSUS IN EXTREMELY LOW BIRTH WEIGHT INFANTS?}

doi:10.1136/archdischild-2012-302724.0318

${ }^{1} \mathrm{JH}$ Lee, ${ }^{2} \mathrm{JH}$ Shin, ${ }^{3} \mathrm{KH}$ Park, ${ }^{2} \mathrm{YJ}$ Rhie, ${ }^{1} \mathrm{MS}$ Park, ${ }^{2} \mathrm{BM}$ Choi. 'Department of Pediatrics, Ajou University School of Medicine, Suwon; '2Departement of Pediatrics, Korea University College of Medicine, Ansan-Si; ${ }^{3}$ Department of Pediatrics, CHA Gangnam Medical Center, Seoul, Republic of Korea

Background and amis Optimal management of a patent ductus arteriosus (PDA) is important for improving the clinical outcomes of extremely low birth weight (ELBW) infants. Although it is reported that prophylactic cyclooxygenase inhibitors result in favorable immediate outcomes, not only serious side effect such as nephrotoxicity but also unnecessarily drug exposure without benefit are inevitable. To investigate the predictability of B-type natriuretic peptide (BNP) for early targeted treatment of hemodynamically significant PDA (hsPDA) in ELBW infants.

Methods 73 ELBW infants that underwent echocardiographic evaluation and plasma BNP measurement after birth were enrolled. 31 infants developed hsPDA (HsPDA group) and 42 infants didn't develop hsPDA (nPDA group).

Results BNP levels of HsPDA group were significantly higher than those of nPDA group at 24 hours of age (921[318-2133] vs. 152 [91-450], pg/mL) but not different at 12 hours of age. BNP levels at 24 hours of age were significantly correlated with the magnitudes of the ductal shunt but not significant at 12 hours of age. The area under the receiver operator characteristic curve of BNP levels for prediction of hsPDA at 12 and 24 hours of age was 0.584 and 0.830 , respectively. At the cutoff BNP level of $200 \mathrm{pg} / \mathrm{mL}$ and $900 \mathrm{pg} / \mathrm{mL}$ at 24 hours of age, the sensitivity was $83.9 \%$ and $54.8 \%$ and the specificity was $61.9 \%$ and $95.2 \%$, respectively.

Conclusions BNP levels at 24 hours of age can be used as a guide for early targeted treatment of hsPDA and avoid the unnecessary use of cyclooxygenase inhibitors in ELBW infants.

\section{THE EFFECT OF MILRINONE INFUSION ON CEREBRAL PERFUSION IN NEONATES WITH CONGENITAL HEART DISEASE PRIOR TO CARDIAC SURGERY}

doi:10.1136/archdischild-2012-302724.0319

1,2MO Bianchi, 'PY Cheung, 'E Phillipos, ${ }^{2} \mathrm{~A}$ Aranha-Netto, ${ }^{1} \mathrm{C}$ Joynt. 'Pediatrics, University of Alberta, Edmonton, AB, Canada; ${ }^{2}$ Pediatrics, UNICAMP, Campinas, Brazil

Background Milrinone has been increasingly used in the postoperative care of neonates with congenital heart disease (CHD) for its inotropic and vasodilatation properties. When used as a preoperative cardiovascular supportive agent, cerebral hemodynamic effects of milrinone have not been studied.

Methods From June 2008 to July 2010, 18 neonates with CHD and treated with milrinone before cardiac surgery were prospectively enrolled. Milrinone $(0.75 \mathrm{mcg} / \mathrm{kg} / \mathrm{min})$ was given according to institutional guidelines and neonates $(n=1)$ requiring additional vasoactive agents were excluded. Using Doppler studies, cardiac output (CO), flow velocity $(\mathrm{Vm})$ and Resistive Index (RI) for anterior and middle cerebral arteries were assessed and analyzed blindly at specific time-points after milrinone administration.

Results Seventeen neonates were studied (gestation: 39.5[36-41] weeks; birth-weight: 3350[2590-4230]g). Hypoplastic left heart syndrome was the most common CHD. Milrinone was commenced at day $1-7$ (88\% on day 1$)$ of life, with heart rate $141 \pm[S D] 14 \mathrm{bpm}$, mean blood pressure $44 \pm 6 \mathrm{mmHg}$ and $\mathrm{CO} 479 \pm 147 \mathrm{ml} / \mathrm{min} / \mathrm{kg}$ at baseline. At $6 \mathrm{~h}, 24 \mathrm{~h}$ and $48 \mathrm{~h}, \mathrm{CO}$ was significantly increased by $23 \%, 20 \%$ and $28 \%$ from pre-treatment baseline, respectively, with increased anterior $(22 \%, 35 \%, 38 \%)$ and middle $(34 \%, 36 \%, 35 \%)$ cerebral $\mathrm{Vm}$. There were no significant changes in heart rate 